Louisiana State University

LSU Digital Commons

Faculty Publications

Department of Chemistry

3-30-2006

\title{
Supramolecular structures in nanocomposite multilayered films
}

\author{
Eduard A. Stefanescu \\ Louisiana State University \\ Avinash Dundigalla \\ Louisiana State University \\ Vincent Ferreiro \\ Université de Lille \\ Elena Loizou \\ Louisiana State University \\ Lionel Porcar \\ National Institute of Standards and Technology
}

See next page for additional authors

Follow this and additional works at: https://digitalcommons.Isu.edu/chemistry_pubs

\section{Recommended Citation}

Stefanescu, E., Dundigalla, A., Ferreiro, V., Loizou, E., Porcar, L., Negulescu, I., Garno, J., \& Schmidt, G. (2006). Supramolecular structures in nanocomposite multilayered films. Physical Chemistry Chemical Physics, 8 (14), 1739-1746. https://doi.org/10.1039/b517880k

This Article is brought to you for free and open access by the Department of Chemistry at LSU Digital Commons. It has been accepted for inclusion in Faculty Publications by an authorized administrator of LSU Digital Commons. For more information, please contact ir@lsu.edu. 


\section{Authors}

Eduard A. Stefanescu, Avinash Dundigalla, Vincent Ferreiro, Elena Loizou, Lionel Porcar, loan Negulescu, Jayne Garno, and Gudrun Schmidt 


\title{
Supramolecular structures in nanocomposite multilayered films $\dagger$
}

\author{
Eduard A. Stefanescu, ${ }^{a}$ Avinash Dundigalla,${ }^{a}$ Vincent Ferreiro, ${ }^{b}$ Elena Loizou, ${ }^{a c}$ \\ Lionel Porcar, ${ }^{c}$ Ioan Negulescu, ${ }^{a}$ Jayne Garno ${ }^{a}$ and Gudrun Schmidt ${ }^{* a}$
}

\author{
Received 16th December 2005, Accepted 8th February 2006 \\ First published as an Advance Article on the web 28th February 2006 \\ DOI: $10.1039 / \mathbf{b 5 1 7 8 8 0 k}$
}

\begin{abstract}
We investigate the multilayered structures of poly(ethylene)oxide/montmorillonite nanocomposite films made from solution. The shear orientation of a polymer-clay network in solution combined with simultaneous solvent evaporation leads to supramolecular multilayer formation in the film. The resulting films have highly ordered structures with sheet-like multilayers on the micrometer length scale. The polymer covered clay platelets were found to orient in interconnected blob-like chains and layers on the nanometer length scale. Inside the blobs, scattering experiments indicate the polymer covered and stacked clay platelets oriented in the plane of the film. The polymer is found to be partially crystalline although this is not visible by optical microscopy. Atomic force microscopy suggests that the excess polymer, which is not directly adsorbed to the clay, is wrapped around the stacked platelets building blobs and the polymer also interconnects the polymer-clay layers. Overall our results suggest the re-intercalation of clay platelets in films made from exfoliated polymer-clay solutions as well as the supramolecular order and hierarchical structuring on the nanometer, via micrometer to the centimeter length scale.
\end{abstract}

\section{Introduction}

Novel physical properties of soft and bulk polymer nanocomposite materials are strongly dependent on the structures at the nanometer length scale and the supramolecular organization of these nanostructures. ${ }^{1-4}$ The presence of the nanoparticle and the interaction of the polymer with the particle as well as the particle orientation in an aqueous precursor phase may lead to a variety of ordered composite materials in the bulk or film. ${ }^{2,5}$ Anisotropic clay particles promote supramolecular organization $^{2,6}$ similar to other systems such as liquid crystalline polymers, ${ }^{7}$ surfactants, ${ }^{8}$ block copolymers ${ }^{9-11}$ and peptides. ${ }^{12}$ Depending on the concentration of polymer and clay, the polymer type and molecular weight, the clay size, shape and surface chemistry, one may generate a variety of dispersions, ${ }^{13,14}$ solutions, ${ }^{15}$ smart gels, ${ }^{16,18}$ shake gels, ${ }^{19,20}$ glues ${ }^{16-18}$ and gum-like gels ${ }^{16-18,21,22}$ all of which may be excellent

\footnotetext{
${ }^{a}$ Louisiana State University, Department of Chemistry, Baton Rouge, LA 70803-1804,USA. E-mail: gudrun@LSU.edu; Fax: 225578 3458; Tel: 2255787375

${ }^{b}$ Laboratoire de Structure et Propriétés de l'Etat Solide, UMR CNRS 8008, Université des Science et Technologie de Lille, Villeneuve d'Ascq, France

${ }^{c}$ National Institute of Standards and Technology, Gaithersburg, MD 20899, USA

$\dagger$ According to ISO 31-8, the term "molecular mass" has been replaced by "relative molecular mass", symbol $M_{\mathrm{r}}$. Thus, if this nomenclature and notation were to be followed, one would write, $M_{\mathrm{r} w}$, instead of the historically conventional $\mathrm{Mw}$ for the mass average molecular weight and it would be called the "mass average relative molecular mass". The conventional notation rather than the ISO notation has been employed for this publication. Certain equipment and instruments or materials are identified in this paper in order to adequately specify the experimental details. Such identification does not imply recommendation by the National Institute of Standards and Technology nor does it imply the materials are the best available for the purpose.
}

precursors for hierarchically structured bulk materials. ${ }^{23,24}$ Some of these soft and hard nanocomposite materials are sensitive to external stimuli including light, temperature, $\mathrm{pH}$, salt concentration, shear, pressure and electric fields, leading to novel properties that attract much scientific and technological interest. ${ }^{21,24}$

To examine the polymer-clay interactions, a combination of methods is advisable. Among them, microscopy and scattering are techniques for studying structure and providing a measure of size, shape and interfacial polymer conformation. Recent advances in ultra small angle scattering techniques offer advantages and complementary information. ${ }^{25}$ Rheology and mechanical testing may nicely distinguish between properties of chemically ${ }^{21}$ vs physically cross-linked polymerclay materials. ${ }^{17,18}$ Since shear can influence both the macroscopic texture and the orientation of the anisotropic particle on the nanometer length scale, it is helpful to combine the above mentioned techniques and correlate changes in the mechanical properties with changes in structure on different length scales.

In this work we are interested in the fabrication of hierarchically ordered model poly(ethylene)oxide (PEO) nanocomposites. The exfoliation, intercalation and aggregation of clays in PEO nanocomposites has been extensively studied in the past. ${ }^{26-32}$ The dispersion of clay platelets in polymer nanocomposite films can be achieved using both solution and melt fabrication techniques. ${ }^{28,29,33}$ Polymers such as PEO are able to intercalate and exfoliate clays directly from the polymer melt as well as promote the exfoliation process in aqueous solution. If the clay and polymer are hydrophilic, no clay surface modification is necessary for complete exfoliation of clay in aqueous solution. The products obtained from simple melt and solution intercalation of PEO into clays are less ordered but may have identical structures. 
Hydrophilic polymers such as PEO strongly adsorb to natural montmorillonite as well as synthetic laponite clay. The amount of polymer adsorbed to the clay is controlled by the layer charge density on the clay. ${ }^{31}$ In PEO-montmorillonite nanocomposites studied in literature the heterogeneous nucleation of PEO competes with the PEO coordination to sodium ions, which inhibits PEO crystallinity. ${ }^{34-36}$ The addition of salt and clay to polymer electrolytes such as PEO leads to a class of materials that offer great potential in electrochemical applications. ${ }^{37}$ By inducing anisotropy over large length scales and via supramolecular ordered structures, new ways are found for fabricating scientifically and technologically novel materials with enhanced mechanical, optical properties and conductivity. ${ }^{35,36}$

The supramolecular organization of clay platelets in polymer nanocomposite films can be achieved using solution fabrication techniques such as self-assembly, a variety of layer-by-layer approaches ${ }^{38}$ as well as the Langmuir-Blodgett method. ${ }^{39-41} \mathrm{We}$ are interested in the fabrication of highly oriented PEO-montmorillonite and PEO-laponite multilayered films from the corresponding highly viscous aqueous gels and solutions. Our previous rheological ${ }^{18}$ and small-angle neutron scattering (SANS) ${ }^{17}$ studies on these PEO-clay solutions and gels have investigated the shear orientation and structure in solution. In solution the polymer and clay form 3-D networks. ${ }^{16}$ The adsorbed PEO polymer is strongly attached to the clay and the excess polymer that is not adsorbed is stabilizing the polymer clay network. The excess polymer in solution usually leads to formation of polymer-rich phases that penetrate the network-like polymer-clay phases. For "non-oriented" bulk materials these disordered phases have been observed before in literature. ${ }^{28,29}$ For multilayered films made from the corresponding gels and solutions we are the first to explore the spontaneous multilayer formation and the supramolecular organization of clay platelets into unusual and highly ordered structures.

\section{Experimental}

We have prepared viscoelastic solutions of the natural smectite type clay, montmorillonite, cloisite NA+ (CNA), (Southern Clay Products), ${ }^{42}$ and poly(ethylene-oxide) (PEO), purchased from Polysciences Inc., $\left(M_{\mathrm{w}}=1 \times 10^{6} \mathrm{~g} \mathrm{~mol}^{-1}, M_{\mathrm{w}} / M_{\mathrm{n}} c a\right.$ : $1.5, R_{\mathrm{g}} \approx 100 \mathrm{~nm}$ in $\left.\mathrm{H}_{2} \mathrm{O}\right) .{ }^{43}$ The CNA clay produces an opaque suspension of predominantly "exfoliated" platelets (no peaks at high $q$ in SANS) ${ }^{44}$ that range on average in size from $c a .70$ to $150 \mathrm{~nm}$ across and are $c a .1 \mathrm{~nm}$ thick (atomic force microscopy). Several $<10 \mathrm{~nm}$ and $c a$. $>1-2 \mu$ large platelets can also be observed, thus the reported polydispersity of $30 \%$ for the platelet diameter ${ }^{42}$ may not be accurate for every type of natural clay, but dependent on the batch and the source. Polydispersity also depends on the treatment and purification of CNA clay, especially if the purification process removes the smaller platelets or the largest ones. Discrepancies between measured sizes for natural clays from the same supplier are known. ${ }^{44,45}$

Multilayered films were prepared from solution via gel/ solution exfoliation while exfoliated and stable solutions were obtained for a particular polymer clay ratio, $\mathrm{pH}$ and ionic strength as described before. ${ }^{16-18,46}$ Here multilayered films are discussed that have been prepared from an aqueous solution containing mass fractions of $3 \%$ clay and $2 \%$ PEO at ambient temperature. The solution $\mathrm{pH}$ and ionic strength were controlled by the addition of $\mathrm{NaOH}(\mathrm{pH}=9-10)$ and $\mathrm{NaCl}(1 \mathrm{mM})$, respectively. Using a $\mathrm{pH} \gg 10$ or $\mathrm{pH} \ll 9$ in solution leads to chemical breakdown of the clay over time. ${ }^{47}$ Gels were spread onto glass slides layer-by-layer and dried at $25{ }^{\circ} \mathrm{C}$ in desiccators and under vacuum. While one spread and dried film ( $c a$. 3-7 $\mu$ thick) already produces multilayers as examined by SEM, the film thickness of one spread film is too small to be cut and thoroughly investigated by scattering and microscopic techniques used by us. Therefore we use a layer by layer spreading and drying technique to obtain thicker films for better investigation. SEM was repeatedly used to examine the interface between the spread layers but no interface could be detected indicating intermixing of spread layers.

Reproducible results were obtained when using and comparing two preparation techniques: (i) a simple spreading device can be used that guarantees the same thickness of each spread layer and (ii) the films are spread manually with a blade. Multilayered films containing $c a .60 \%$ of CNA clay and $40 \%$ of PEO polymer (by mass fraction) were obtained (CNA60\%-PEO40\%). More detailed film preparation is described elsewhere. ${ }^{48}$ The shear-orientation combined with the drying procedure as well as control of the film thickness is absolutely necessary to obtain the highly ordered multilayers; simply drying the film is not sufficient. The film structure of the CNA60\%-PEO40\% films has been found to be independent of the spreading direction.

SANS measurements were preformed on the $30 \mathrm{~m}$ SANS NG7 instrument at the Center for Neutron Research (NCNR), National Institute of Standards and Technology (NIST). ${ }^{49}$ In a standard $y$-beam configuration, the incident beam is perpendicular to the spread direction of the film and the SANS intensity is obtained in the $x-z$ plane. In the $z$-beam configuration, the incident beam is parallel to the spread direction of a $1 \mathrm{~mm}$ thick PEO-clay film (see Fig. 1 and 2). The neutron beam in $z$-beam configuration provided SANS intensities in the $x-y$ plane. The primary contrast in the SANS experiment is between the silicate and PEO. This allows SANS experiments

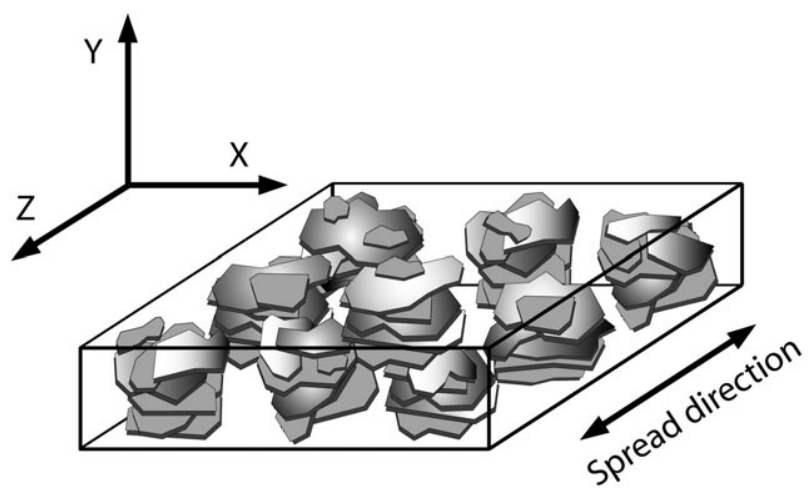

Fig. 1 A physical picture of general clay platelet orientation in a multilayered polymer nanocomposite film is shown as well as the definition of planes. 


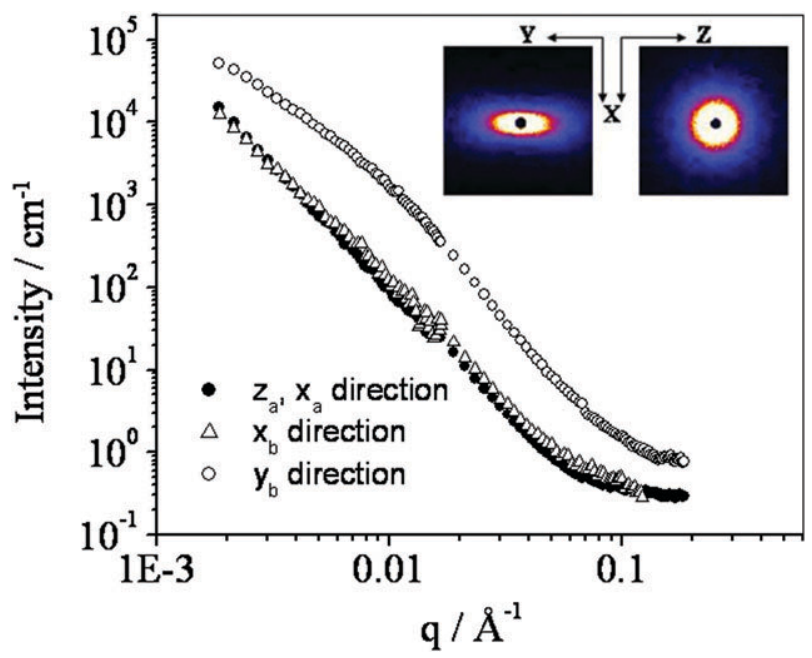

Fig. 2 SANS intensity as averaged in 10 degree sectors for all three directions in space. 2D SANS spectra from a $c a .1 \mathrm{~mm}$ thick multilayered film obtained in the $x-z$ plane and in the $x-y$ plane.

to detect the overall orientation of the clay platelets in a polymer matrix (Fig. 2).

Sample preparation for the AFM measurements included cryo-ultramicrotom slicing (Leica ultracut with FC4 from Reichert-Jung) and freeze fracture. Samples were cut at $-120{ }^{\circ} \mathrm{C}$, below the PEO glass transition temperature $\left(T_{\mathrm{g}}=\right.$ $\left.-55{ }^{\circ} \mathrm{C}\right)$. The images were recorded with a Nanoscope IIIa Dimension 3100 (Veeco Instruments). ${ }^{50}$ As reported in a previous paper, ${ }^{50} \mathrm{AFM}$ "phase imaging" can be used to distinguish clay particles from the PEO matrix due to their difference in mechanical properties. AFM images in the $x-z$ plane were acquired in air using a deflection type scanner (PicoSPM) from Molecular Imaging (Tempe, AZ).

SEM experiments were performed using a Cambridge 260 Stereoscan Electron Microscope. Many fractures in all three planes were investigated and only representative images are presented. A TA 2920 MDSC instrument has been used for differential scanning calorimetry (DSC) measurements. Samples of $2.5-10 \mathrm{mg}$ have been subjected to analysis using a heating rate of $5{ }^{\circ} \mathrm{C} \mathrm{min}^{-1}$. The collected data have been normalized to a PEO content of $1.0 \mathrm{mg}$. Thermogravimetric analyses (TGA) have been performed in nitrogen atmosphere with a heating rate of $5{ }^{\circ} \mathrm{C} \mathrm{min}{ }^{-1}$ using a TA 2950 thermo balance. The X-ray diffraction measurements were done using a Siemens-Bruker D5000 X-ray Diffractometer with the $\mathrm{Cu}$ $\mathrm{K} \alpha$ radiation, of $1.54 \AA$. Duplicate measurements on all instruments show excellent reproducibility with a relative uncertainty of $c a .5 \%$.

\section{Results and discussion}

The solution structure and fabrication conditions strongly influence the morphology of the multilayered dried films. ${ }^{24}$ In solution the adsorbed PEO polymer is strongly attached to the clay and the excess polymer that is not adsorbed is stabilizing the polymer-clay network. ${ }^{28,29,51}$ The predominant orientation of CNA platelets in solution is with the flow and with the surface normal along the velocity direction. ${ }^{52}$ The orientation of CNA platelets in the dried film is expected to be in the film plane (Fig. 1). A simple physical picture of clay platelet orientation in the multilayered films as well as the definition of planes is shown in Fig. 1. The orientation of the clay platelets can be deduced from the SANS results (Fig. 2). The isotropic SANS pattern in the $x-z$ plane and the anisotropy observed in the $x-y$ plane confirms the orientation of the platelets to be with the surface normal perpendicular to the film plane ( $x-z$ plane). From the 2-D SANS patterns in $x-z$ and $x-y$ direction, the intensity as a function of $q$ can be calculated in all three directions in space. Anisotropy is observed over at least 2 orders of magnitude in $q$ which indicates orientation of clay platelets over the whole $q$ range is detected. A change in slope between $0.001-0.01$ and $0.01-0.1$ reciprocal Angstroms or the hint of a shoulder that is observed around $q=0.01$ reciprocal Angstroms $(2 \pi / q \approx 60 \mathrm{~nm})$ may be correlated with the $c a .56 \mathrm{~nm}$ thickness of layers detected by microscopy. Intensities in $x$ and $z$ directions as well as the $x$ directions from both configurations overlap as expected. Since the SANS is averaged over the sample volume, the 2-D SANS patterns shown in Fig. 2 look similar to SANS patterns from other oriented nanocomposites studied in the past. ${ }^{23,24}$ However results from microscopy show very unusual and unexpected structures.

The polymer-clay morphoplogy and texture orientation as displayed from representative atomic force microscopy (AFM) is illustrated in Fig. 3 and 4. Compared to what is usually found in literature on polymer nanocomposite orientation, our results show an unusual and unexpected 3-D ordered and layered structure of blob-like chains and layers. The orientation of individual CNA clay platelets with an average diameter of $c a .70-150 \mathrm{~nm}$ and a thickness of $1 \mathrm{~nm}$ does not easily explain the presence of ordered layers of elongated "blobs" (Fig. 3 and 4). The average blob thickness is $c a .56 \mathrm{~nm}$ and the average blob length is $c a .100 \mathrm{~nm}$ (Fig. 4) while a chain of blobs can be very long. The $56( \pm 16) \mathrm{nm}$ blob chain thicknesses ( $y$-direction) correspond to polymer wrapped clay stack layers as seen from the side (the blob may contain several platelets). X-Ray diffraction experiments discussed later confirm the presence of stacked clay platelets. Although in solution the clay platelets were found to be exfoliated (no peaks in SANS and SAXS) in the film, the platelets re-intercalate and order supramolecularly. The high polydispersity of natural montmorillonite CNA clay (average size $70-150 \mathrm{~nm}$ ) leads to heterogeneities and more defects in orientation compared to the low disperse synthetic laponite LRD clay $(30 \pm 5 \mathrm{~nm}$ diameter platelets). ${ }^{24}$ Multilayered films shown in Fig. 4a and $\mathrm{b}$, strongly reflect the differences in polydispersity of CNA $v s$ LRD clay. The average $x$-direction correlation length observed from several AFM images (such as Fig. 3 and 4) is ca. $100( \pm 20) \mathrm{nm}$ per blob for CNA60\%-PEO40\%. This blob length corresponds to an average clay diameter for CNA platelets which is around $100 \mathrm{~nm}$ (Fig. 4). For LRD60\%-PEO40\% shown in Fig. 4b the correlation length of the blobs observed is much smaller, more uniform and on the order of 30-60 nm.

If we assume that $c a .100 \mathrm{~nm}$ is the correct average CNA clay platelet diameter then we may wonder what happened 
a)

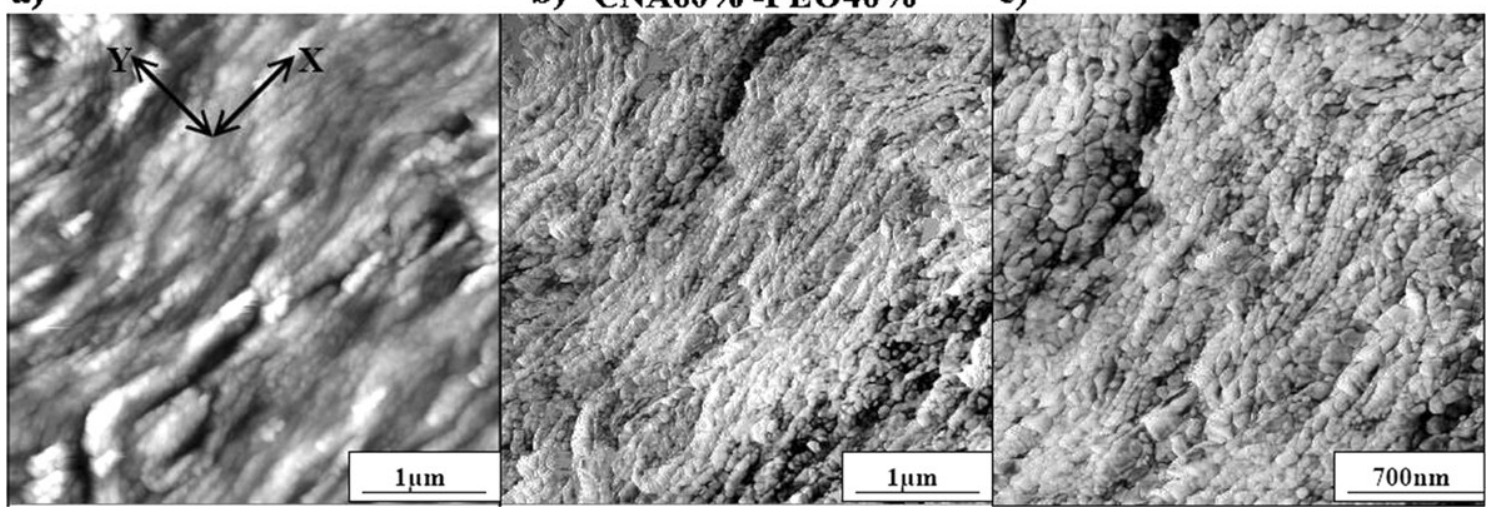

Fig. 3 AFM (a) height image and (b)-(c) phase images from the $x-y$ plane sections of CNA60\%-PEO40\% multilayered films.

with the few large platelets that are $>1 \mu$ in size? AFM from pure and diluted CNA solutions suggests the presence of few large platelets $>1 \mu$. It is possible that many of the large platelets are broken down during the sample preparation process and those few remaining form large defects that are not shown with AFM but may be visualized by optical microscopy. An alternate interpretation suggests that it is possible for the larger polymer covered platelets to be arranged between the blob-like chains and layers since the length of some of these layers is on the order of several $1000 \mathrm{~nm}$. This interpretation is supported by the nm size layered structures we have observed for films at higher salt concentration studied by us in the past. ${ }^{46}$ Here large amounts of excess PEO may cover up any blob-like chains and lead to a different type of layered structure. ${ }^{46}$ Due to higher polymer concentrations neither height nor phase imaging can distinguish between individual platelets at CNA40\%-PEO60\% concentrations studied in the past. ${ }^{46}$ Although the -2D SANS data for both, the CNA40\%-PEO60\% films studied in the past ${ }^{46}$ and the CNA60\%-PEO40\% films presented here look qualitatively very similar, the local morphology such as the interconnected blobs $v s$ layers, is very different.

To better understand the film structure formation we need to know the polymer clay interactions in solution. In solution the clay particles can only adsorb a maximum amount of polymer until all the clay surfaces are covered. ${ }^{51}$ The polymer and the clay build a network-like structure that is interpenetrated by a sub-network of interconnecting pores containing excess polymer and water. ${ }^{51}$ Since the polymer adsorbed clay is completely exfoliated in solution (no peaks in diffraction patterns from solution) this solution-structure must collapse, reorder and re-intercalate into blob-like chains during the film formation process. The more or less uniform blob size observed in the film is highly reproducible and must be related in some way to the network-like structure in solution.

For synthetic laponite clay the absorbed polymer layer has been measured before to be $c a .1 .5 \mathrm{~nm}$ on each face. ${ }^{51}$ In the film the excess polymer is wrapped around the stacked laponite clay platelets forming $30-60 \mathrm{~nm}$ blobs (Fig. 4b). ${ }^{24}$ Montmorillonite (CNA) clay does the same, just that the blobs observed are more polydisperse and elongated due to larger aspect ratio (ca. $100 \mathrm{~nm}$ long and $56 \mathrm{~nm}$ thick blobs) (Fig. 4a). Our preliminary results from solutions also showed that the platelets within a polymer-clay network are interconnected over the edge more than over the face. ${ }^{51}$ We hypothesize that when the network is stretched, and the solvent evaporates simultaneously, the network collapses, the clay platelets reintercalate and the edge to edge connection in solution may

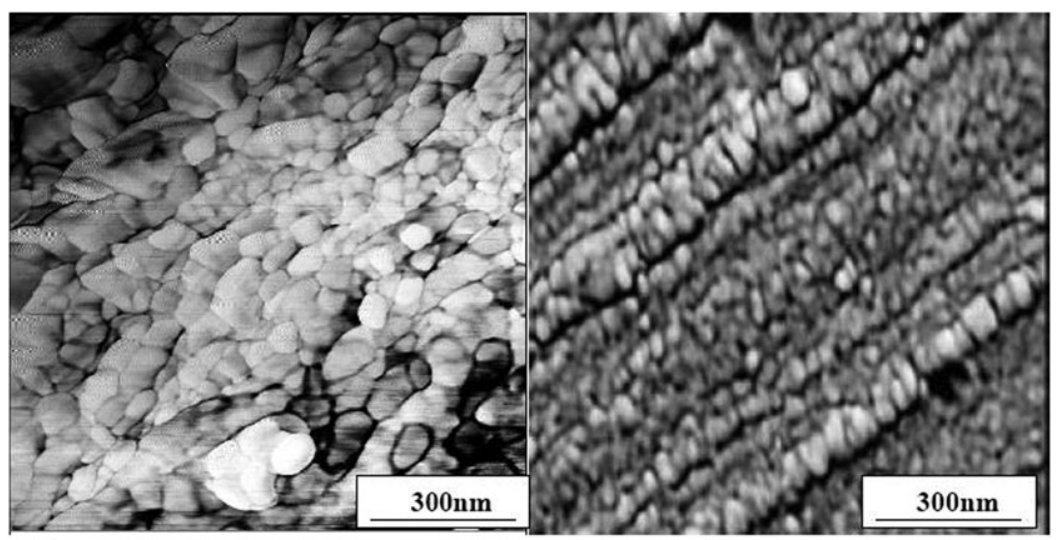

a) $\mathrm{CNA60 \% -PEO40 \%}$

b) LRD60\%-PEO40\%

Fig. 4 (a) AFM images from the $x-y$ plane sections of multilayered films for CNA60\%-PEO40\% and (b) LRD60\%-PEO40\%, both phase images. For (a) and (b) the clay concentration is high enough as to distinguish individual or bundles of clay particles. 

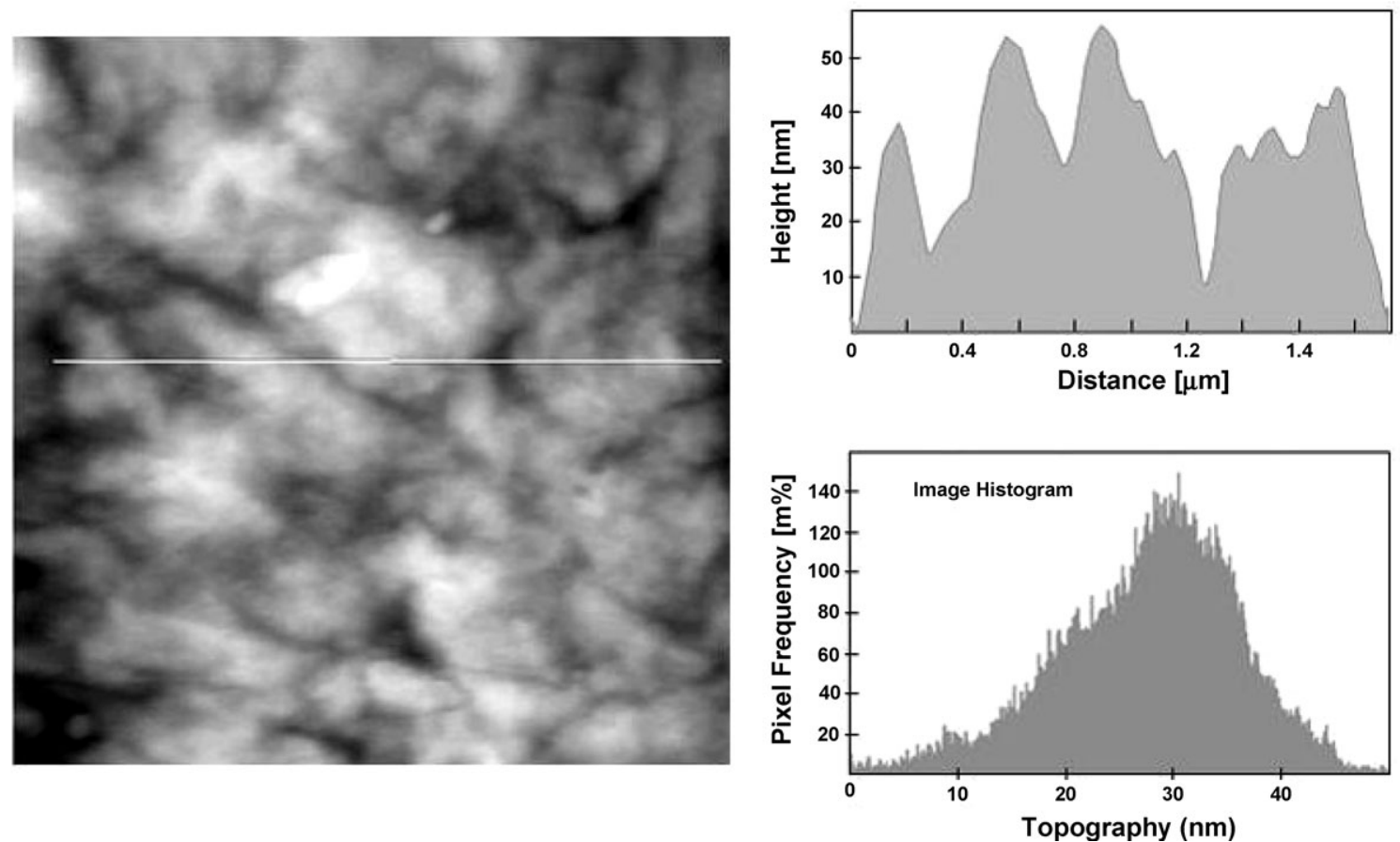

Fig. 5 A representative AFM image is shown from the $x-z$ plane section of a multilayered film for CNA60\%-PEO40\%. Cursor profile and height histogram are also shown. No layered structure is visible.

favor the formation of blob-like chains and sheets in the film. The sliding of already existing blob chains and sheets of a sheared but not completely dried film may also influence the observed layered structures which are very similar to shear oriented liquid crystalline lamellar phases. As for the surface structure of the layers, AFM from representative samples in the $x-z$ plane is shown in Fig. 5. Cursor profile and height histogram are also shown. No layered structure is observed.

Scanning electron microscopy (SEM) is used to determine the film morphology on the micron length scale (Fig. 6). As mentioned previously, the aqueous CNA-PEO solutions can be described as interconnected networks. ${ }^{52}$ When the sample is shear-oriented and the solvent evaporates simultaneously, the network collapses which leads to layered film structures that can be observed on several length scales. In the $x-y$ plane of the films (Fig. 6), SEM was used to examine whether an interface exists between individual spread layers. Similar to other polymer-clay multilayered films that we have studied before $^{46}$ no boundaries between spreading layers (each 3-8 $\mu \mathrm{m})$ could be detected, indicating substantial intermixing of spread layers. Nevertheless a highly ordered and layered structure of the films is observed in the $x-y$ plane, while no layers are observed in the $x-z$ plane. The layered texture observed in the $x-y$ direction is not uniform and is calculated to have an average dimension of $d_{\mathrm{SEM}} \approx 60-70 \mathrm{~nm}$ per layer (Fig. 6c). Even though the exact shear rate during the

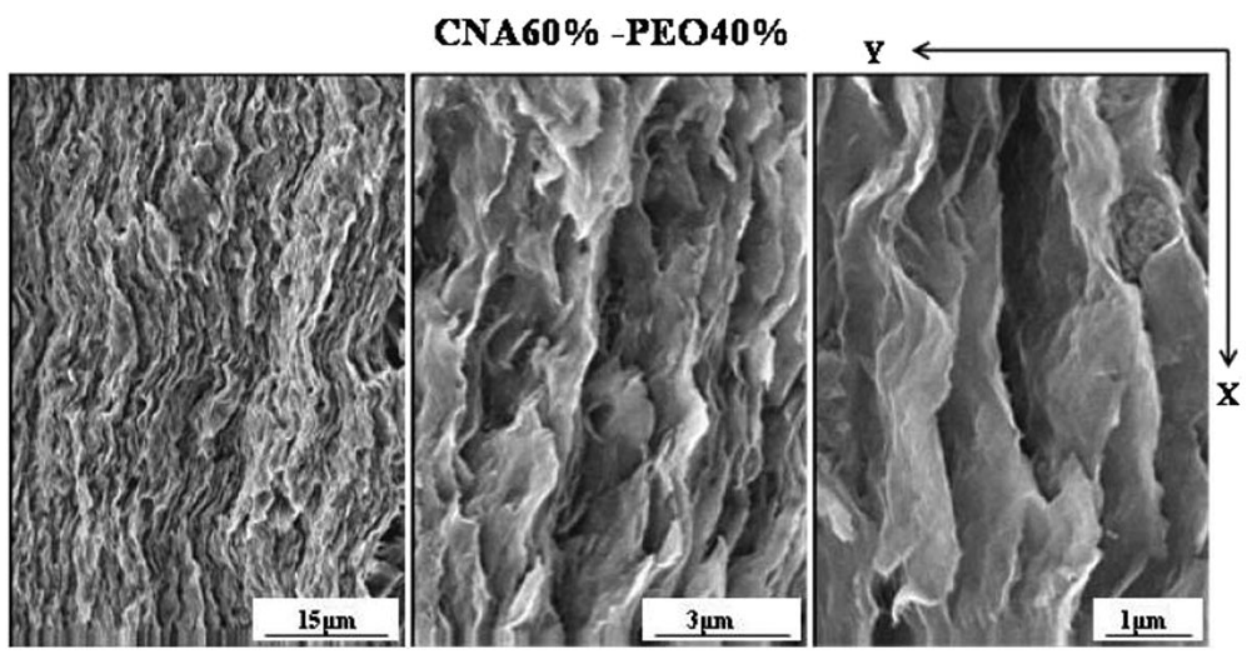

Fig. 6 SEM images of freeze fractured $x-y$ plane surfaces of CNA60\%-PEO40\% multilayered films at different magnifications. A distinct layered structure is visible. 
spreading process cannot be controlled, SEM shows high reproducibility in data. The film structure has also been found to be independent of the spreading direction.

According to AFM measurements each of the $c a$. $60-70 \mathrm{~nm}$ thick layers observed by SEM corresponds to the blob-like chains and layers that are on average $56 \mathrm{~nm}$ thick (Fig. 3 and 4). Reference PEO films made from pure PEO solutions with the same salt concentration as the CNA60\%-PEO $40 \%$ films showed no layered structures suggesting that the addition of salt to pure PEO solutions does not lead to any layer formation.

Analysis of 10 different sample areas $\left(2 \times 2 \mu \mathrm{m}^{2}\right)$ gave an average RMS roughness measurement of $20 \mathrm{~nm}$, with values ranging from $12-29 \mathrm{~nm}$. On a micrometer to centimeter length scale, the CNA60\%-PEO40\% films presented here look different from the CNA40\%-PEO60\% films studied in the past. ${ }^{46}$ The lower polymer concentration used here leads to a more open structure with less interconnected layers in the $x-y$ plane (Fig. 6) and no layers in the top surfaces ( $x-z$ plane, see AFM). The CNA60\%-PEO40\% films presented here also look very different from the LRD $60 \%$-PEO $40 \%$ films studied in the past. ${ }^{24}$ At the same polymer, clay and salt concentrations, the larger CNA platelets ( $c a .100 \mathrm{~nm}$ ) lead to less oriented micrometer size layers compared to the smaller LRD platelets $(c a .30 \mathrm{~nm})$. This is counterintuitive since we expect large CNA particles to align more preferentially than the smaller ones at a similar concentration. The origin of these effects is unclear but must be related to the shear alignment and relaxation of clay platelets in solution. Future work will investigate these effects in more detail. Inspection of several SEM images suggests that the fractured CNA nanocomposite film does not break parallel to the layers while the LRD nanocomposite film does. ${ }^{24}$

The microscopic structure of the multilayered films was characterized by polarized optical microscopy, which showed differences in birefringence in each plane (Fig. 7). From our experience with other nanocomposite films such as LRD $60 \%-\mathrm{PEO} 40 \%$ at the same salt concentration ${ }^{24}$ we would expect to see no birefringence in the $x-z$ plane and strong birefringence in the $x-y$ plane which is predominantly coming from clay platelets that are hierarchically ordered on all length scales. For CNA-PEO films at higher polymer concentration and different salt concentration we would expect and observe birefringence in both planes resulting from both the polymer and the clay. ${ }^{46}$ When comparing films ideally one would like to limit the number of parameters being altered in the films made from solutions. However this is often impossible due largely to the very complicated phase diagrams of these complex systems in aqueous solutions that may require change of parameters (such as salt, excess polymer) to prevent the solution/dispersion from phase separation. ${ }^{52}$

The $x-z$ plane (see Fig. 1 for plane, Fig. 7 for optical microscopy) for CNA60\%-PEO40\% films shows only few speckles which are due to the birefringence observed from predominantly single large clay platelets and clusters. With increasing temperatures in a range from 25 to $200{ }^{\circ} \mathrm{C}$ the overall birefringence is only somewhat reduced probably due to melting of any oriented polymer. To the eye no significant differences in birefringence are visible. Observation of the

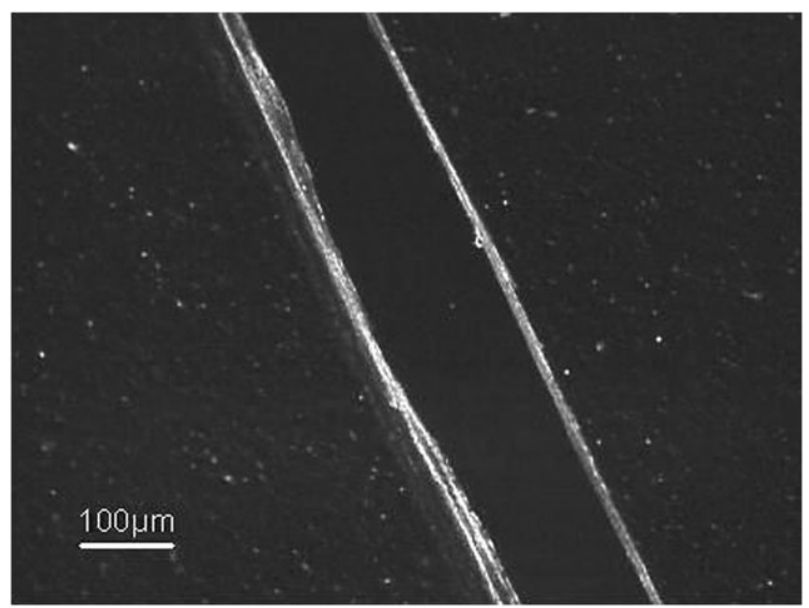

Fig. 7 Representative optical microscopy image from a nanocomposite film with a cut surface. Crossed polarizers are used. A small section of a one layered film was removed to expose the $x-y$ plane. The film is shown at room temperature after being heated and cooled. Birefringent speckles and birefringence of the $x-y$ plane do not disappear at high temperatures. This film has been heated and cooled to remove any birefringence coming from additional shear effects during the scratching or cutting of the film.

exposed edge of the film, the $x-y$ plane, shows a highly birefringent pattern even after annealing for $1 \mathrm{~h}$ at $200{ }^{\circ} \mathrm{C}$. The total birefringence of the film is dominated by the orientation of the clay platelets and the polymer within the sample. For CNA60\%-PEO40\% studied here the polymer contribution to the total birefringence is either small or "not visible" to the eye, suggesting that optical microscopy is not the best method for detecting PEO crystallites that may be confined between the layers.

Differential scanning calorimetry (DSC): While the crystallinity of various bulk PEO nanocomposites at low clay concentrations has been studied extensively in the past, ${ }^{30,31,53}$ here we focus on supramolecularly oriented and anisotropic materials at high clay concentrations. The polymer and clay composition of our multilayered CNA $60 \%-\mathrm{PEO} 40 \%$ films is confirmed by DSC experiments. The pure CNA clay as obtained from Southern clay has $c a .2 \%$ of "impurities" based on the dry material. These are detected in TGA thermograms as a weight loss in nitrogen atmosphere at around $150{ }^{\circ} \mathrm{C}$.

DSC data shown in Fig. 8 are normalized to $1 \mathrm{mg}$ content of PEO; therefore the enthalpic change for PEO from the nanocomposite is corrected to $97 \mathrm{~J} \mathrm{~g}^{-1}$ (i.e., $39: 0.4 \approx 97$ ). If we consider the pure PEO to be $100 \%$ crystalline $^{43}$ then our results from the nanocomposite films show that $52 \%$ of the total polymer content is crystalline and $48 \%$ is amorphous (Fig. 8). These results suggest that the high clay concentration is sufficient to suppress $48 \%$ of the PEO crystallization in the film. We expected that the CNA clay adsorbed polymer could be amorphous since it is confined to the clay surface and cannot move easily. The excess polymer would then form most of the crystalline phase. However qualitatively none of the PEO crystallites are visible with optical microscopy, thus PEO crystallites may be either too small as to be detected by optical 


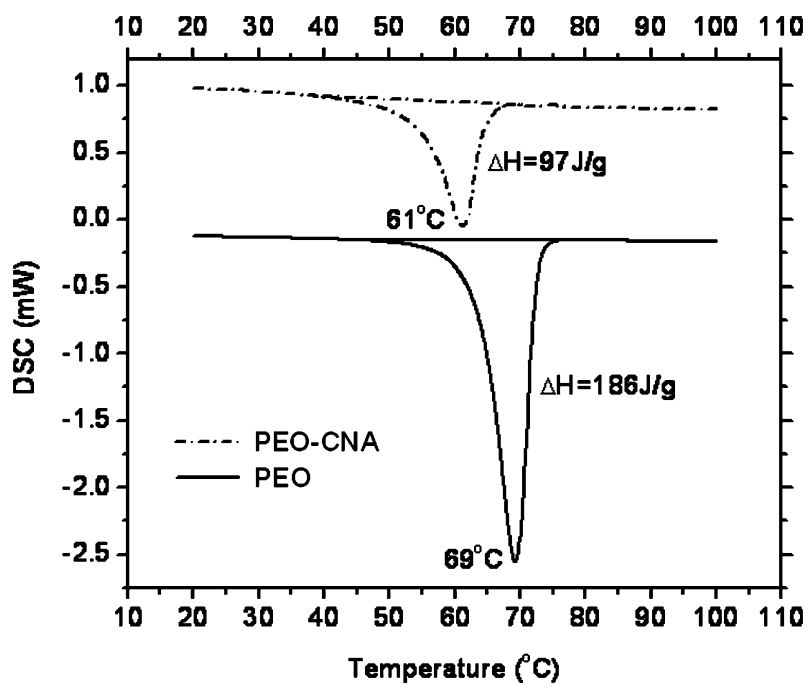

Fig. 8 Normalized DSC plots for crystalline melting of the pure PEO polymer $(y=$ DSC data) and of the polymer nanocomposite CNA60\%-PEO40\% $(y=$ DSC $+1.1 \mathrm{~mW}$ shifted $)$. Heating rate, $5{ }^{\circ} \mathrm{C} \mathrm{min}{ }^{-1}$ in nitrogen.

microscopy or there may be polymer crystallinity confined within the clay layers.

It has been found in previous work that montmorillonite clay can adsorb $c a .0 .3 \mathrm{~g}$ PEO per g of CNA clay. For a film with CNA60\%-PEO40\% the adsorbed amount is then calculated to be $45 \%$ of the total polymer content. ${ }^{28-30}$ This is more or less in agreement with our DSC results which give $48 \%$ for amorphous polymer. Since the presence of salt may influence the crystal formation of PEO as has been observed by other groups in the past ${ }^{35,36}$ we believe that discrepancies between the $45 \%$ calculated from literature and the $48 \%$ from our DSC data may result from the presence of salt. At the same time, examining the DSC traces shown in Fig. 8 one may notice also that the melting temperature of PEO crystals in the clay composite was lowered significantly as compared to that of the pure PEO sample (consider for example the peak temperatures), pointing to an inhibitory effect of salt during PEO crystallization in the clay matrix. The difference in melting temperature however is too large to come from the presence of salt alone but may also be attributed to differences in crystalline structures. ${ }^{34}$ These interesting peculiarities will be investigated in more detail in the future.

Differences in crystallinity of the CNA60\%-PEO $40 \%$ films presented here $(52 \%)$ compared to the same composition LRD $60 \%-\mathrm{PEO} 40 \%$ films studied in the past (near $0 \%)^{24}$ show that clay size dependence leads to unexpected but reproducible phenomenology at the nanoscale.

X-ray diffraction patterns of CNA60\%-PEO40\% films and from pure CNA and pure PEO reference samples are shown in Fig. 9. Although the polymer-clay solutions from which the nanocomposite films are made, are completely exfoliated (no peaks visible). XRD confirms that the dried multilayered films are highly structured. The XRD reflections predominantly correspond to the PEO intercalated clay suggesting the presence of stacked layers. Peaks from crystallized PEO are probably present but are not visible due to the high intensity

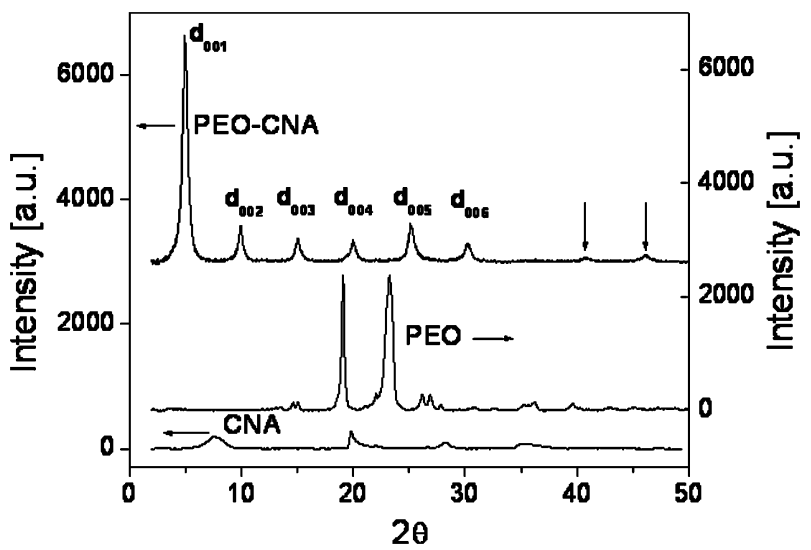

Fig. 9 X-ray diffraction patterns of CNA60\%-PEO40\% film (shifted +3000 intensity units), of the pure CNA and the pure PEO reference samples.

peaks coming from the clay. Here DSC is the better method for measuring PEO crystallinity. The first peak in XRD corresponds to a $d$-spacing of $d=17.8 \AA$, a result in agreement with what has been found in literature for other polymer nanocomposites. ${ }^{29}$ The XRD pattern of CNA60\%-PEO40\% looks similar to other melt intercalated PEO-montmorillonite nanocomposites (even those at other clay content) found in literature. ${ }^{29}$ Similar to the SANS data shown in Fig. 2, the XRD averages over the sample volume, thus both SANS and XRD do not show the hierarchical arrangements as microscopy does.

The big difference between the nanocomposites obtained via melt and solution intercalation of previous studies ${ }^{28,29}$ and the nanocomposites studied here is this supramolecular order and hierarchical structuring we observe. On the nanometer length scale a high degree of order is reflected in the XRD pattern but AFM is necessary to visualize the supramolecular structure. Compared to work done in the past our nanocomposite films have highly anisotropic structure from the nanometer, via micrometer to the $\mathrm{cm}$ length scale while many previously reported nanocomposites have only local ordered structures. Overall our results suggest the re-intercalation of clay platelets in films made from exfoliated polymer-clay solutions as well as the possibility to supramolecular order and hierarchical structure. The structure and properties of our multilayered nanocomposite films may provide a useful route in the preparation of novel materials such as anisotropic solid state electrolytes with enhanced ionic conductivity in only one direction.

\section{Acknowledgements}

We acknowledge the support of NIST in providing the neutron research facilities used in this work. We also acknowledge financial support in part from an NSF-CAREER award, DMR 0348884. The authors thank J. M. Gloaguen (LSPES, Lille, France) for performing cryo-ultramicrotome film preparations. We acknowledge the use of the Socolofsky Microscopy Center at Louisiana State University. We thank the reviewers for useful comments. 


\section{References}

1 B. M. Novak, Adv. Mater., 1993, 5, 422-433.

2 G. Schmidt and M. M. Malwitz, Curr. Opin. Colloid Interface Sci., 2003, 8, 103-108.

3 M. Antonietti, Colloid Chemistry I, Springer, Berlin, 2003, vol. 226.

4 R. Krishnamoorti and R. A. Vaia, Polymer Nanocomposites, ACS, Washington DC, 2002, vol. 804.

5 R. A. Vaia and E. P. Gianellis, MRS Bull., 2001, 62, 394- 401.

6 J. C. P. Gabriel, F. Camerel, B. J. Lemaire, H. Desvaux, P. Davidson and P. Batail, Nature, 2001, 413, 504-508.

7 G. Schmidt, S. Muller, C. Schmidt and W. Richtering, Rheol. Acta, 1999, 38, 486-494.

8 G. Schmidt, S. Muller, P. Lindner, C. Schmidt and W. Richtering, J. Phys. Chem. B, 1998, 102, 507-513.

9 H. A. Klok and S. Lecommandoux, Adv. Mater., 2001, 13, 1217-1229.

10 G. Schmidt, W. Richtering, P. Lindner and P. Alexandridis, Macromolecules, 1998, 31, 2293-2298.

11 K. Almdal, K. A. Koppi, F. S. Bates and K. Mortensen, Macromolecules, 1992, 25, 1743-1751.

12 K. Rajagopal and J. P. Schneider, Curr. Opin. Struct. Biol., 2004, 14, 480-486.

13 H. A. Baghdadi, H. Sardinha and S. R. Bhatia, J. Polym. Sci., Part $B, 2005,43,233-240$.

14 N. N. Herrera, J. M. Letoffe, J. L. Putaux, L. David and E. Bourgeat-Lami, Langmuir, 2004, 20, 1564-1571.

15 J. Liu and H. Hoffmann, Colloid Polym. Sci., 2004, 283, 24-32.

16 G. Schmidt, A. I. Nakatani, P. D. Butler and C. C. Han, Macromolecules, 2002, 35, 4725-4732.

17 G. Schmidt, A. I. Nakatani, P. D. Butler, A. Karim and C. C. Han, Macromolecules, 2000, 33, 7219-7222.

18 G. Schmidt, A. I. Nakatani and C. C. Han, Rheol. Acta, 2002, 41, 45-54.

19 J. Zebrowski, V. Prasad, W. Zhang, L. M. Walker and D. A. Weitz, Colloids Surf., A, 2003, 213, 189-197.

20 D. C. Pozzo and L. M. Walker, Colloids Surf., A, 2004, 240, 187-198.

21 K. Haraguchi and T. Takehisa, Adv. Mater., 2002, 14, 1120-1124.

22 K. Haraguchi, T. Takehisa and S. Fan, Macromolecules, 2002, 35, 10162-10171.

23 A. Bafna, G. Beaucage, F. Mirabella and S. Mehta, Polymer, 2003, 44, 1103-1115.

24 A. Dundigalla, S. Lin Gibson, V. Ferreiro, M. M. Malwitz and G. Schmidt, Macromol. Rapid Commun., 2005, 26, 143-149.

25 S. R. Bhatia, Curr. Opin. Colloid Interface Sci., 2005, 9, 404-411.

26 P. Aranda, Y. Mosqueda, E. Perez-Cappe and E. Ruiz-Hitzky, $J$. Polym. Sci., Part B, 2003, 41, 3249-3263.

27 P. Aranda and E. Ruiz-Hitzky, Appl. Clay Sci., 1999, 15, 119-135.

28 J. H. Wu and M. M. Lerner, Chem. Mater., 1993, 5, 835-838.
29 R. A. Vaia, S. Vasudevan, W. Krawiec, L. G. Scanlon and E. P. Giannelis, Adv. Mater., 1995, 7, 154-156.

30 D. J. Chaiko, Chem. Mater., 2003, 15, 1105-1110.

31 J. Bujdak, E. Hackett and E. P. Giannelis, Chem. Mater., 2000, 12, 2168-2174.

32 G. Lagaly, Appl. Clay Sci., 1999, 15, 1-9.

33 G. Lagaly and S. Ziesmer, Adv. Colloid Interface Sci., 2003, 100, $105-128$.

34 K. E. Strawhecker and E. Manias, Chem. Mater., 2003, 15, 844-849.

35 Z. Gadjourova, Y. G. Andreev, D. P. Tunstall and P. G. Bruce, Nature, 2001, 412, 520-523.

36 Z. Gadjourova, D. M. Marero, K. H. Andersen, Y. G. Andreev and P. G. Bruce, Chem. Mater., 2001, 13, 1282-1285.

37 L. Edman, A. Ferry and M. M. Doeff, J. Mater. Res., 2000, 15, 1950-1954.

38 Z. Y. Tang, N. A. Kotov, S. Magonov and B. Ozturk, Nat. Mater., 2003, 2, 413-418.

39 B. van Duffel, R. A. Schoonheydt, C. P. M. Grim and F. C. De Schryver, Langmuir, 1999, 15, 7520-7529.

40 G. Decher, Science, 1997, 277, 1232-1237.

41 G. Decher and J. Schlenoff, Multilayer Thin Films: Sequential Assembly of Nanocomposite Materials, VCH Verlagsgesellschaft, 2003.

42 Polydispersity is estimated to be $c a .30 \%$ by the supplier.

43 J. Brandup, E. H. Immergut and E. A. Grulke, Polymer Handbook, John Wiley and Sons, New York, 1999.

44 D. L. Ho, R. M. Briber and C. J. Glinka, Chem. Mater., 2001, 13, 1923-1931.

45 H. J. M. Hanley, C. D. Muzny, D. L. Ho and C. J. Glinka, Langmuir, 2003, 19, 5575-5580.

46 M. M. Malwitz, A. Dundigalla, V. Ferreiro, P. D. Butler, M. C. Henk and G. Schmidt, Phys. Chem. Chem. Phys., 2004, 6, 2977-2982.

47 D. W. Thompson and J. T. Butterworth, J. Colloid Interface Sci., 1992, 151, 236-243.

48 M. M. Malwitz, S. Lin-Gibson, E. K. Hobbie, P. D. Butler and G. Schmidt, J. Polym. Sci. Part B, 2003, 41, 3237-3248.

49 C. J. Glinka, J. G. Barker, B. Hammouda, S. Krueger, J. J. Moyer and W. J. Orts, J. Appl. Crystallogr., 1998, 31, 430-445.

50 V. Ferreiro and G. Coulon, J. Polym. Sci., Part B, 2004, 42, 687-701.

51 E. Loizou, P. D. Butler, L. Porcar, Y. Talmon, E. Kesselman and G. Schmidt, Macromolecules, 2005, 38, 2047-2049.

52 M. M. Malwitz, P. D. Butler, L. Porcar, D. P. Angelette and G. Schmidt, J. Polym. Sci., Part B, 2004, 42, 3102-3112.

53 N. Ogata, S. Kawakage and T. Ogihara, Polymer, 1997, 38, 5115-5118. 